\title{
Proteomic and Immunological Identification of Antigens from Ticks with Artificial Antiserum
}

\author{
Ke Zhang ${ }^{1}$, Shuai Liu ${ }^{2}$, Qiu Chen ${ }^{1}$, Yu Wang ${ }^{1}$, Li Liu ${ }^{1}$, Bingjie Li ${ }^{1}$, Kai Ma ${ }^{1}$, \\ Xiaoya Wei ${ }^{1}$, Aijun $\mathrm{Li}^{3}$ and Junyan $\mathrm{Li}^{2 *}$ \\ ${ }^{1}$ Medical College, Pingdingshan University, Pingdingshan 467000, China \\ ${ }^{2}$ National Institute of Occupational Health and Poison Control, Chinese Center for \\ Disease Control and Prevention, Beijing 100000, China \\ ${ }^{3}$ Pingdingshan Center for Disease Control and Prevention, Pingdingshan 467000, China
}

Ke Zhang and Shuai Liu contributed equally to this work.

\begin{abstract}
A B S T R A C T
We aimed to screen novel tick antigens using two-dimensional gel electrophoresis (2-DE) followed by western blot analysis (WB) with artificial antiserum. A total of 105 Haemaphysalis longicornis $(H$. longicornis) ticks were selected to prepare protein samples. Some of them were used for 2-DE, and the others were used to immunize mice to collect tick antiserum for WB. Then, 2-DE followed by WB and MALDI-TOF was carried out to screen and identify tick antigens. We identified 19 protein spots representing 12 ORFs: 4 from ticks and 8 from cattle. Among the the 4 ORFs, Tropomyosin, Elongation factor 1-alpha (EF-1 $\alpha)$ and Protein Disulfide Isomerase-2 (PDI-2) have been reported as antigens and even vaccine candidate proteins in ticks or other pathogens but not in H. longicornis. Few reports have described dual specificity phosphatase 3 (DUSP-3) in parasites as an antigen. We successfully screened four potential antigens and found that DUSP-3 is a potential novel antigen for ticks.
\end{abstract}

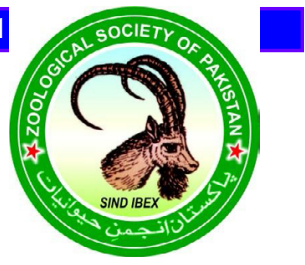

Article Information

Received 16 January 2019

Revised 30 March 2019

Accepted 14 March 2020

Available online 15 May 2020

Authors' Contribution

JL and KZ designed the study. QC,

YW and XW collected the samples.

SL, LL, BL, KM and AL performed

the experimental work. $\mathrm{KZ}$ analysed

the data and wrote the manuscript.

Key words

2-DE-WB, Antigen, Artificial

antiserum, Tick

\section{INTRODUCTION}

$\mathrm{T}$ icks are blood-feeding ectoparasites with a worldwide distribution and transmit bacterial, parasitic and viral pathogens that cause diseases in humans and animals. Tick vaccines offer the advantages of being cost-effective and environmentally friendly alternatives to other tick control measures, with the dual effects of reducing tick infestation and preventing ticks from transmitting pathogens (Contreras et al., 2016). The success of tick vaccines depends on the detection, identification, and functional investigation of antigenic proteins that target specific proteins that play pivotal roles in tick life processes, such as blood feeding and digestion processes (Kröber et al., 2007). 2-DE followed by WB and MALDI-TOF is an effective technique for antigenic protein separation, detection and identification, and antiserum plays a key role in the binding of antigenic proteins.

Usually, to generate antiserum, live ticks are allowed to bite the host, and the antiserum is collected for detection of tick antigenic proteins. During blood sucking, saliva is secreted into the host by the ticks. The saliva interacts with

\footnotetext{
* Corresponding author: lijunyan688@126.com 0030-9923/2020/0005-1801 \$ 9.00/0

Copyright 2020 Zoological Society of Pakistan
}

the immune system of the host, which generates specific antibodies targeting tick saliva. In WB, the antigens are screened with specific antibodies that bind the antigens. This approach is limited because antigens from saliva are limited in number; thus, so is the antiserum from the host bitten by the ticks. However, candidate vaccine antigens can be obtained from sources other than saliva; for example, Bm86 is a tick gut glycoprotein (Rand et al., 1989). Thus, if the antigens used to immunize the host are from the whole tick body, the antiserum from the host will represent a more comprehensive range of proteins than those from the saliva.

In this study, we extracted proteins from whole ticks, mixed them with adjuvant, and immunized mice to prepare antisera. We then screened tick antigenic proteins using 2-DE-WB and MALDI-TOF.

\section{MATERIALS AND METHODS}

\section{Tick collection}

Ticks were collected from two local cattle grazing on a mountain near the city of Pingdingshan. The ticks were identified to the species level by morphology (Murrell et $a l ., 2001)$. The ticks were frozen in liquid nitrogen and immediately stored at $-80^{\circ} \mathrm{C}$ until use.

A total of 105 ticks were collected from two cattle 
and contained a mix of fed, unfed, and partially fed adults and nymphs. All ticks were identified as $H$. longicornis. Protein samples from whole tick bodies were extracted, some for 2-DE and 2-DE-WB and the rest for immunizing mice.

\section{Protein sample preparation}

All the ticks we collected were washed with precooled PBS containing penicillin/streptomycin. Then, the ticks were placed in a mortar with liquid nitrogen for grinding. The ticks were ground into powder without obvious particles while liquid nitrogen was added continuously to the mortar. Then, the powder was dissolved in $800 \mu \mathrm{l}$ of ALK lysis buffer (containing $2 \mathrm{M}$ thiourea, 2\% CHAPS, 7 $\mathrm{M}$ urea, $10 \mathrm{mM}$ DTT, $1 \mathrm{mM}$ PMSF, and $20 \mathrm{mM}$ Tris) and sonicated (ultrasonic treatment $5 \mathrm{~s}$, interval $10 \mathrm{~s}$, power $120 \mathrm{w}, 99$ cycles, performed in ice water until clear and bright). Then, the lysates were centrifuged at $15,000 \mathrm{rpm}$ for 30 minutes at $4^{\circ} \mathrm{C}$. Then, $250 \mu \mathrm{l}$ of the supernatant and $1 \mathrm{ml}$ of precooled acetone $\left(4^{\circ} \mathrm{C}\right)$ were mixed and stored at $-20^{\circ} \mathrm{C}$ for one night to precipitate proteins. Finally, the precipitated proteins were centrifuged at $20,000 \mathrm{rpm}$ for 30 minutes at $4^{\circ} \mathrm{C}$, collected, and placed on clean filter paper for natural acetone volatilization. Then, the proteins were dissolved in ALK lysis buffer and quantitated using a BCA Protein Assay Kit (Thermo Fisher Scientific Pierce). Finally, the protein samples were stored at $-80^{\circ} \mathrm{C}$ until 2-DE and antiserum preparation.

\section{Generation of tick antisera in mice}

Freund's adjuvant is mineral oil that is emulsified together with a solution of an antigen to form a waterin-oil emulsion. The water-in-oil emulsion continuously releases the antigen and stimulates the immune response, resulting in enhanced cellular and humoral antibody responses. Complete Freund's Adjuvant (CFA) contains whole or pulverized heat-killed mycobacteria, while Incomplete Freund's Adjuvant (IFA) does not. Although the use of CFA can result in severe side effects, and thus alternatives to CFA should be used whenever possible, no comparable alternatives are known to exist (Jackson and Fox, 1995). To generate high-titer tick antisera in mice, proteins from whole ticks mixed with CFA for the initial immunization and IFA for a subsequent immunization and booster immunization were used to immunize mice by subcutaneous injection at multiple sites. Seven-week-old female BALB/c mice (SPF) were purchased from Sibeifu Animals Biotechnology Co., Ltd. (Beijing, China). The mice were bred in the Experimental Animal Room at the Chinese Centre for Disease Control and Prevention, Beijing. The mouse feeding was performed in individual ventilated cages (IVCs); the conditions were a temperature of $20-23^{\circ} \mathrm{C}$, humidity of $40-70 \%$, illumination of $15-20 \mathrm{~lx}$, air change rate of $\geq 15 \mathrm{~T} / \mathrm{h}$, and light/dark cycle of $10 / 14$ $\mathrm{h}$. The mice were free to drink and eat. The drinking water was prepared by UV irradiation after reverse osmosis filtration. Irradiation-sterilized Co60 mouse feed was purchased from Beijing Keaoxieli Feed Co., Ltd.

After five days of adaptive feeding, when the mice were 8 weeks old and approximately $22 \mathrm{~g}$ in weight, the initial immunization began. There was a twoweek interval between the initial and the subsequent immunization and between the subsequent immunization and the booster immunization. Equal amounts of adjuvant were mixed with whole tick protein samples, and the mixtures were formulated immediately before application. Subcutaneous injection was performed at five sites. For initial immunization of each mouse, $100 \mu \mathrm{g}$ of whole tick proteins and $100 \mu \mathrm{L}$ of CFA were mixed, and the CFA dose per site was $20 \mu \mathrm{L}$. Our protocol was in conformity with the Guidelines for the Use of Adjuvants in Research. The detailed parameters of the immunization program are shown in Table I. The blank controls were immunized using an equal amount of sterilized physiological saline. Four days after the last subsequent immunization, blood was collected and the serum precipitated naturally at room temperature, and the IgG concentration was tested with an IgG ELISA kit (Biolab, Beijing China, cat. no. 23060077).

\section{Two-dimensional gel electrophoresis (2-DE)}

After the whole tick proteins were prepared, the proteins were separated by 2-DE first, and the methods were similar to those described previously (Zhou et al., 2013). Briefly, each whole tick protein sample $(1500 \mu \mathrm{g})$ was mixed with rehydration buffer (containing $2 \mathrm{M}$ thiourea, $7 \mathrm{M}$ urea, $2 \mu \mathrm{L}$ of IPG buffer, $10 \mu \mathrm{L}$ of $1 \mathrm{M}$ DTT and $4 \%$ CHAPS). The volume of the mixture was $450 \mu \mathrm{L}$. Then, the mixture was loaded onto an IPG strip (Immobiline 17 $\mathrm{cm}, \mathrm{pH}$ 4-7, from Bio-Rad, USA) without introducing air bubbles, and isoelectric point focusing was carried out by an Ettan IPGphor 3 apparatus (GE Healthcare, Sweden). The total VH was approximately 110,000 . Then, the strip was equilibrated for reduction of the proteins in $8 \mathrm{~mL}$ of SDS equilibration buffer (with $50 \mathrm{mM}$ Tris- $\mathrm{HCl} \mathrm{pH} 8.8,6$ $\mathrm{M}$ urea, $30 \%$ glycerol, $2 \%$ SDS, $160 \mu \mathrm{L}$ of 1 M DTT and $0.002 \%$ bromophenol blue), and then the proteins were separated by SDS-PAGE (13\%). 2-DE was performed in duplicate three times. Three of the gels were dyed with Coomassie brilliant blue R-250 for spot excision, whereas the other gels were used for WB.

Two-dimensional gel electrophoresis immunoblotting (2-DE-WB)

After the tick antisera were prepared in mice, the 
other gels were used for WB. The methods were similar to those described previously (Zhao et al., 2015). In brief, the proteins in the gel were transferred to nitrocellulose membranes. Then, the membrane was blocked in TBST (TBS buffer containing 1\% Tween-20 and 5\% skimmed milk). Then, for antigen-antibody (primary antibody) binding, the membrane was incubated with tick antiserum diluted $1: 250(\mathrm{v} / \mathrm{v})$ at $4^{\circ} \mathrm{C}$ overnight. Then, the membrane was washed with TBST buffer. For antibody-anti-antibody binding, the membrane was incubated with secondary antibody (biotinylated goat anti-mouse IgG antibody) for two hours. The membrane was washed with TBST. Finally, an ECL Western Blot kit (Beijing CoWin Biotech, Beijing, China) was used to detect antigens on the membrane. The positive spots on the 2-DE gels were then excised and subjected to MALDI-TOF analysis according to the results of WB.

\section{Identification of antigenic proteins (MALDI-TOF/MS)}

The protein spots corresponding to mouse antisera identified were excised manually from the 2-DE gels. The methods of MALDI-TOF were similar to those described previously (Pazouki et al., 2009). In brief, the protein spots we excised were cleaned with Milli-Q water twice and decolorized with 50\% methanol until they were colorless. After dehydration, trypsin digestion, and peptide extraction, the peptides were dissolved in resolving solution. Then, the samples were repeatedly aspirated and spotted, dried at room temperature, and analyzed by MALDI-TOF-MS on a mass spectrometer (Bruker Dalton, Germany). FlexAnalysis (Bruker Dalton) software was used to filter the signal baseline peak and distinguish the signal peak. BioTools (Bruker Dalton) software was used to search for peptides and proteins in the Universal Protein databases.

\section{RESULTS}

\section{Mouse tick antisera}

During the whole process of immunization, the mice were in good condition. The injection sites of mice were without skin ulceration, local abscesses or tissue sloughing, and the mice were without (obvious) pain and distress. After three immunizations, mouse blood was collected. The serum was precipitated naturally at room temperature, and the concentration of $\mathrm{IgG}$ antibody was tested. The IgG concentration was, on average, $893.75 \mu \mathrm{g} / \mathrm{ml}$ for the mice immunized with tick whole-body proteins, which was far higher than the average of $226 \mu \mathrm{g} / \mathrm{ml}$ for those immunized with equal amounts of sterilized physiological saline.
Comparative proteomic analysis by 2-DE and 2-DE-WB

Protein spots were distributed throughout the 2-DE gel (Fig. 1a), with a concentration of spots at PI 7 and a smeared band at $70 \mathrm{kDa}$. 2-DE and $\mathrm{WB}$ revealed many protein spots clustered at $>40 \mathrm{kDa}$ in the gel. Ultimately, nineteen spots were screened after 2-DE, WB and 2-DE matching (Fig. 1a, 1b).

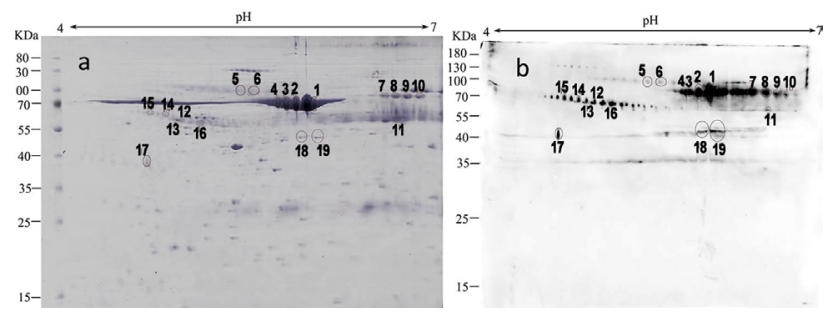

Fig. 1. Identification of antigenic proteins from the whole body of the tick H. longicornis (Acari: Ixodidae) (a) 2-DE gel stained for whole tick proteins; (b) Spots selected for MALDI-TOF/MS analysis. Note: The circles in the figure show the antigenic proteins sent for MALDI-TOF analysis.

\section{Identification of antigenic proteins from $\mathrm{H}$. longicornis}

We found nineteen protein spots that were subjected to MALDI-TOF analysis. A protein with several matched peptides (score >50) the established peptide mass fingerprint database was considered significant (Ma et al., 2009). In the peptide mass fingerprint analysis, the mass results of protein spots $1,12,13,17-19$ showed the highest correlations with proteins from Ixodoidea, with peptide sequence coverages of $15-64 \%$ and sequence scores of $72-$ 896. In addition, protein spots 1-12 and 14-19 showed the highest correlations with proteins from the host cattle, with peptide sequence coverages of $3-76 \%$ and sequence scores of 64-4161 (Table II).

\section{DISCUSSION}

Currently, vector control by immunological approaches is the most important strategy for the prevention and control of major infectious diseases throughout the world. However, the success of vaccines depends on the specific proteins we screen, specifically whether these proteins play key roles in pathogenic processes or not (Kröber et al., 2007). 2-DE followed by WB and MALDITOF was an effective technique for antigenic protein separation, detection and identification. In this technique, antigens were bound by specific antibodies from antisera and identified. To identify antigens other than those from only tick saliva, we generated antisera by using whole tick body proteins to immunize mice. Ultimately, we selected 19 protein spots and identified 12 antigenic proteins: four antigenic proteins from ticks and eight from cattle blood. 
Table I. Immunization program.

\begin{tabular}{lllll}
\hline Immunization program & Interval & Dose $(\boldsymbol{\mu g} \mathbf{g} \mathbf{m o u s e})$ & Adjuvant & Route \\
\hline Initial immunization & 5 days after adaptive feeding & 100 & CFA & Back, subcutaneous \\
Subsequent immunization & 2 weeks after initial immunization & 100 & IFA & Back, subcutaneous \\
Booster immunization & 2 weeks after subsequent immunization & 80 & IFA & Back, subcutaneous \\
Blood collection & Four days after booster immunization & ---- & ----- & ---- \\
\hline
\end{tabular}

CFA means Complete Freund's Adjuvant, and IFA means Incomplete Freund's Adjuvant. Dose means the whole tick protein dose in units of $\mu \mathrm{g} / \mathrm{mouse}$.

Table II. Summary of antigenic proteins identified in the whole bodies of $\boldsymbol{H}$. longicornis by MALDI-TOF.

\begin{tabular}{|c|c|c|c|c|c|c|c|}
\hline & $\begin{array}{l}\text { Spot } \\
\text { no. }\end{array}$ & $\begin{array}{l}\text { Protein ID (Gen- } \\
\text { Bank accession no.) }\end{array}$ & Description & $\begin{array}{l}\text { Protein } \\
\text { score }\end{array}$ & $\begin{array}{l}\text { Sequence } \\
\text { coverage }(\%)\end{array}$ & $\begin{array}{l}\text { No. of peptides } \\
P<0.05\end{array}$ & $\begin{array}{l}\text { Calculated } \\
\text { MW/PI }\end{array}$ \\
\hline \multirow[t]{6}{*}{ Tick } & 1 & JAP76203.1 & $\begin{array}{l}\text { Dual-specificity phosphatase } \\
3\end{array}$ & 72 & $42 \%$ & 10 & $29732 / 5.98$ \\
\hline & 12 & BAF63671.1 & Protein disulfide isomerase-2 & 127 & $15 \%$ & 6 & $57270 / 4.94$ \\
\hline & 13 & BAF63671.1 & Protein disulfide isomerase-2 & 284 & $48 \%$ & 24 & $57270 / 4.91$ \\
\hline & 17 & AAN05633.1 & Tropomyosin & 632 & $64 \%$ & 25 & $32853 / 4.69$ \\
\hline & 18 & EEC13271.1 & Elongation factor 1-alpha & 896 & $17 \%$ & 5 & $51048 / 9.18$ \\
\hline & 19 & JAA71972.1 & Elongation factor 1-alpha & 525 & $16 \%$ & 3 & $51048 / 9.18$ \\
\hline \multirow[t]{18}{*}{ Bovine } & 1 & ALBU_BOVIN & Serum albumin & 593 & $48 \%$ & 24 & $71244 / 5.98$ \\
\hline & 2 & ALBU_BOVIN & Serum albumin & 563 & $42 \%$ & 25 & $71244 / 5.84$ \\
\hline & 3 & ALBU_BOVIN & Serum albumin & 599 & $45 \%$ & 25 & $71244 / 5.76$ \\
\hline & 4 & ALBU_BOVIN & Serum albumin & 510 & $28 \%$ & 15 & $71244 / 5.69$ \\
\hline & 5 & G5E513_BOVIN & Uncharacterized protein & 174 & $17 \%$ & 7 & $50623 / 5.40$ \\
\hline & 6 & G5E5T5_BOVIN & Uncharacterized protein & 142 & $21 \%$ & 10 & $43013 / 5.50$ \\
\hline & 7 & TRFE_BOVIN & Serotransferrin & 526 & $40 \%$ & 26 & $79870 / 6.55$ \\
\hline & 8 & TRFE_BOVIN & Serotransferrin & 639 & $42 \%$ & 24 & $79870 / 6.64$ \\
\hline & 9 & TRFE_BOVIN & Serotransferrin & 609 & $36 \%$ & 24 & $79870 / 6.73$ \\
\hline & 10 & G3X6N3_BOVIN & Serotransferrin & 588 & $37 \%$ & 21 & $79783 / 6.83$ \\
\hline & 11 & ALBU_BOVIN & Serum albumin & 64 & $5 \%$ & 5 & $71244 / 6.65$ \\
\hline & 12 & ITIH4_BOVIN & $\begin{array}{l}\text { Inter-alpha-trypsin inhibitor } \\
\text { heavy chain } \mathrm{H} 4\end{array}$ & 285 & $6 \%$ & 6 & $101620 / 4.94$ \\
\hline & 14 & SPA31_BOVIN & Serpin A3-1 & 182 & $22 \%$ & 9 & $46322 / 4.82$ \\
\hline & 15 & SPA32_BOVIN & Serpin A3-2 & 157 & $12 \%$ & 5 & $46322 / 4.71$ \\
\hline & 16 & ITIH4_BOVIN & $\begin{array}{l}\text { Inter-alpha-trypsin inhibitor } \\
\text { heavy chain } \mathrm{H} 4\end{array}$ & 514 & $10 \%$ & 9 & $101620 / 5.08$ \\
\hline & 17 & E1BKT9_BOVIN & Uncharacterized protein & 66 & $3 \%$ & 12 & $334572 / 4.69$ \\
\hline & 18 & ALBU_BOVIN & Serum albumin & 4161 & $76 \%$ & 45 & $71244 / 5.82$ \\
\hline & 19 & ALBU_BOVIN & Serum albumin & 3893 & $72 \%$ & 39 & $71244 / 5.82$ \\
\hline
\end{tabular}

The tropomyosin protein is a salivary protein that has actin regulator activity and plays an important role in allergic reactions against parasites (Ranjbar et al., 2014). This protein has previously been reported to be an antigenic protein in ectoparasites such as Sarcoptes scabiei and the ticks Hyalomma anatolicum and Boophilus annulatus (Nabian et al., 2013; Manjunathachar et al., 2017; Morgan et al., 2016). This is the first time that tropomyosin has 
been identified as an antigenic protein from $H$. longicornis. Protein disulfide isomerases (PDIs) and their homologs play essential roles in the oxidative folding and chaperone-mediated control of proteins in the secretory pathway (Benham, 2012). PDIs from $H$. longicornis expressed primarily in the salivary gland are involved in blood feeding, viability and oocyte development (Liao et al., 2008). Previous studies have shown that PDI is a candidate antigen for vaccines in endoparasites such as Toxoplasma gondii, Leishmania donovani, Besnoitia besnoiti, and even Amblyomma americanum ticks (Radulović et al., 2014; Ma et al., 2009; Wang et al., 2013; Marcelino et al., 2011). This is the first time that PDI-2 has been identified as an antigenic protein in H. longicornis.

Elongation factor 1-alpha (EF-1 $\alpha)$, a GTP-binding protein, is an essential component of the eukaryotic translational apparatus that catalyzes the binding of aminoacyl-transfer RNAs to ribosomes (Tatsuka et al., 1992). It has been found that this protein is a protective antigen or vaccine candidate antigen of several endoparasites, such as Echinococcus granulosus, Cryptosporidium parvum, and Toxoplasma gondii (Margutti et al., 1999; Matsubayashi et al., 2013; Wang et al., 2017, Wang et al., 2015), but not ectoparasites. In this study, EF-l $\alpha$ was identified for the first time as an antigenic protein in H. longicornis.

Dual specificity phosphatases (DUSPs) form a subclass of protein tyrosine phosphatases that can hydrolyze the phosphate ester bond on both a tyrosine and a threonine or serine residue on the same protein (Lyon et al., 2002). DUSPs dephosphorylate threonine and tyrosine residues on mitogen-activated protein kinases (MAPKs) and hence are referred to as MAPK phosphatases (MKPs) (Jeffrey et al., 2007). DUSPs negatively regulate the musculature and development of the whole body in Caenorhabditis elegans (Hu, 2006). Few have been reported to be antigens. In this study, DUSP-3 was identified as an antigenic protein in $H$. longicornis for the first time.

There are some areas in our research that need further improvement. For example, IPG strips of $\mathrm{pH}$ 4-7 were selected in 2-DE, which caused the proteins out of the $\mathrm{pH}$ range of 4-7 to be missed. In addition, proteins with low abundance were likely lost during protein sample preparation. Even if these missed proteins in 2-DE are able to induce the host immune response and generate antibodies, they would not have been detected in WB. However, the antigenic proteins we identified were all in the $\mathrm{pH}$ range of 4-7 and had relatively high abundance. In future research, expanding the range of the IPG strip $\mathrm{pH}$ and reducing the loss of proteins with low abundance will improve the identification of valuable antigenic proteins.
In addition, some proteins from the host were identified, which is not what we intended. In this research, the fed and partially fed ticks contained considerable amounts of undigested blood from the host cattle. In the procedure of antiserum generation, the protein samples made from ticks contained undigested blood. These proteins from undigested blood interacted with the mouse immune system and prompted specific antibody generation. Using 2-DE-WB-MALDI-TOF, antigenic proteins from the host cattle blood were identified, including three inhibitors (inter-alpha-trypsin inhibitor heavy chain $\mathrm{H} 4 \alpha$, Serpin A3-1 and Serpin A3-2), two serum proteins (serum albumin and Serotransferrin), and three proteins with unknown functions (E1BKT9_BOVIN, G5E5T5_BOVIN and G5E513 BOVIN).

\section{CONCLUSIONS}

2-DE followed by WB and MALDI-TOF is a valuable technique for antigen separation and identification. In this study, we screened tick antigens by using antisera from mice immunized with whole tick body proteins, antisera not from animals bitten by ticks. Three antigens we screened have been reported in other tick species or pathogens. Only the screened antigen DUSP-3 has not previously been reported as an antigen. Our results suggest that antisera from animals immunized by proteins extracted from whole ticks are feasible for screening antigens using 2-DE followed by WB and MALDI-TOF.

\section{ACKNOWLEDGEMENTS}

This work was supported by the Henan Science and Technology Plan Projects of China (Nos. 172102310211 and 172102310529) for supporting this study. We are grateful to Guangzhou Fit Gene Biotechnology Co., Ltd., China, for their technical assistance in WB and MALDITOF; to Renfu SHAO and Buyun CUI for their valuable comments on the manuscript.

\section{Ethics approval and consent to participate}

All procedures involving animals in our research were approved and overseen by the Chinese Center for Disease Control and Prevention Institutional Animal Care and Use Committee (No. EAWE-2017-012).

\section{Statement of conflict of interest}

The authors have declared no conflict of interest.

\section{REFERENCES}

Benham, A.M., 2012. The protein disulfide isomerase 
family: key players in health and disease. Antioxid. Redox Signal., 16: 781-789. https://doi.org/10.1089/ ars.2011.4439

Contreras, M., Villar, M., Alberdi, P. and de la Fuente, J., 2016. Vaccinomics approach to tick vaccine development. Meth. Mol. Biol., 1404: 275-286. https://doi.org/10.1007/978-1-4939-3389-1_19

Hu, Y.Y., 2006. Study the biological function of tyrosine/ dual-specifity phosphatase MTM-5 in C. elegans. M. Sc. thesis, Jilin University, Jilin, China

Jackson, L.R. and Fox, J.G., 1995. Institutional policies and guidelines on adjuvants and antibody production. ILAR J., 37: 141-152. https://doi. org/10.1093/ilar.37.3.141

Jeffrey, K.L., Camps, M., Rommel, C. and Mackay, C.R., 2007. Targeting dual-specificity phosphatases: Manipulating MAP kinase signalling and immune responses. Nat. Rev. Drug. Discov, 6: 391-403. https://doi.org/10.1038/nrd2289

Kröber, T. and Guerin, P.M., 2007. In vitro feeding assays for hard ticks, 2007. Trends Parasitol., 23: 445-449. https://doi.org/10.1016/j.pt.2007.07.010

Liao, M., Boldbaatar D., Gong, H., Huang, P., Umemiya, R., Harnnoi, T., Zhou, J., Tanaka, T., Suzuki, H., Xuan, X. and Fujisaki, K., 2008. Functional analysis of protein disulfide isomerases in blood feeding, viability and oocyte development in Haemaphysalis longicornis ticks. Insect. Biochem. Mol. Biol., 38: 285-295. https://doi.org/10.1016/j. ibmb.2007.11.006

Lyon, M.A., Ducruet, A.P., Wipf, P. and Lazo, J.S., 2002. Dual-specificity phosphatases as targets for antineoplastic agents. Nat. Rev. Drug. Discov., 1: 961-976. https://doi.org/10.1038/nrd963

Ma, G.Y., Zhang, J.Z., Yin, G.R., Zhang, J.H., Meng, X.L. and Zhao, F., 2009. Toxoplasma gondii: Proteomic analysis of antigenicity of soluble tachyzoite antigen. Exp. Parasitol., 122: 41-46. https://doi.org/10.1016/j.exppara.2009.01.011

Manjunathachar, H.V., Saravanan, B.C., Kumar, B. and Paramasivam, T., 2017. Expression and determination of immunization dose of recombinant tropomyosin protein of Hyalomma anatolicum for the development of anti-tick vaccine. Indian $J$. Anim. Sci., 87: 275-279.

Marcelino, E., Martins, T.M., Morais, J.B., Nolasco, S., Cortes, H., Hemphill, A., Leitão, A. and Novo, C., 2011. Besnoitia besnoiti protein disulfide isomerase (BbPDI): Molecular characterization, expression and in silico modelling. Exp. Parasitol., 129: 164174. https://doi.org/10.1016/j.exppara.2011.06.012 Margutti, P., Ortona, E., Vaccari, S., Barca, S., Riganò,
R., Teggi, A., Muhschlegel, F., Frosch, M. and Siracusano, A., 1999. Cloning and expression of a cDNA encoding an elongation factor 1beta/ delta protein from Echinococcus granulosus with immunogenic activity. Parasite Immunol., 21: 485-492. https://doi.org/10.1046/j.13653024.1999.00246.x

Matsubayashi, M., Teramoto-Kimata, I., Uni, S., Lillehoj, H.S., Matsuda, H., Furuya, M., Tani, H. and Sasai, K., 2013. Elongation Factor- $1 \alpha$ is a novel protein associated with host cell invasion and a potential protective antigen of Cryptosporidium parvum. J. biol. Chem., 288: 34111-34120. https:// doi.org/10.1074/jbc.M113.515544

Morgan, M.S., Arlian, L.G., Rider, Jr. S.D., Grunwald, Jr. W.C. and Cool, D.R., 2016. A proteomic analysis of Sarcoptes scabiei (Acari: Sarcoptidae). J. med. Ent., 53: 553-561. https://doi.org/10.1093/jme/ tjv247

Murrell, A., Campbell, N.J. and Barker, S.C., 2001. A total-evidence phylogeny of ticks provides insights into the evolution of life cycles and biogeography. Mol. Phylogenet. Evol., 21: 244-258. https://doi. org/10.1006/mpev.2001.1018

Nabian, S., Taheri, M., Fard, R.M. and Aramoon, M., 2013. Identification of tropomyosin and its immunological properties from larvae of cattle tick, Boophilus annulatus. Iran J. Parasitol., 8: 242-248.

Pazouki, N., Sankian, M., Leung, P.T. and KhavariNejad, R.A., 2009. Varasteh Identification of cyclophilin as a novel allergen from Platanus orientalis pollens by mass spectrometry. J. Biosci. Bioeng., 107: 215-217. https://doi.org/10.1016/j. jbiosc.2008.10.016

Radulović, Z.M., Kim, T.K., Porter, L.M., Sze, S.H., Lewis, L. and Mulenga, A., 2014. A 24-48 h fed Amblyomma americanum tick saliva immunoproteome. BMC Genomics, 15: 518. https://doi. org/10.1186/1471-2164-15-518

Rand, K.N., T. Moore, A. Sriskantha, K. Spring, R.Tellam, P. Willadsan and G.S. Cobon, 1989. Cloning and expression of a protective antigen from the cattle tick Boophilus microplus. Proc. natl. Acad. Sci. U.S.A., 86: 9657-9661. https://doi. org/10.1073/pnas.86.24.9657

Ranjbar, M.M., Nabian, Taheri, Nikbakht and Nikpei, 2014. Immunoinformatic survey of Boophilus tick tropomyosin protein. J. Vet. Res., 64: 321-331.

Tatsuka, M., Mitsui, H., Wada, M., Nagata, A., Nojima, H. and Okayama, H., 1992. Elongation factor-1 $\alpha$ gene determines susceptibility to transformation. Nature, 359: 333-336. https://doi.org/10.1038/359333a0 
Wang, H.L., Li, Y.Q., Yin, L.T., Meng, X.L., Guo, M., Zhang, J.H., Liu, H.L., Liu, J.J. and Yin, G.R., 2013. Toxoplasma gondii protein disulfide isomerase (TgPDI) Is a novel vaccine candidate against toxoplasmosis. PLoS One, 8: e70884. https://doi. org/10.1371/journal.pone.0070884

Wang, S., Wang, Y., Sun, X., Zhang, Z., Liu, T., Gadahi, J.A., Hassan, I.A., Xu, L., Yan, R., Song, X. and Li, X., 2015. Protective immunity against acute toxoplasmosis in BALB/c mice induced by a DNA vaccine encoding Toxoplasma gondii elongation factor 1-alpha. BMC Infect. Dis., 15: 448. https:// doi.org/10.1186/s12879-015-1220-5

Wang, S., Zhang, Z., Wang, Y., Gadahi, J.A., Xu. L., Yan, R., Song, X. and Li, X., 2017. Toxoplasma gondii elongation factor 1-alpha (TgEF-1a) is a novel vaccine candidate antigen against toxoplasmosis. Front. Microbiol., 8: 168. https://doi.org/10.3389/ fmicb.2017.00168

Zhao, X.J., Li, L., Kuang, Z., Luo, G. and Li, B., 2015. Proteomic and immunological identification of two new allergens from silkworm (Bombyx mori L.) pupae. Exp. Immunol., 40: 30-34. https://doi. org/10.5114/ceji.2015.50830

Zhou, D.H., Zhao, F.R., Huang, S.Y., Xu, M.J., Song, H.Q., Su, C.L. and Zhu, X.Q., 2013. Changes in the proteomic profiles of mouse brain after infection with cyst-forming Toxoplasma gondii. Parasites Vectors, 6: 1-14. https://doi.org/10.1186/17563305-6-96 